\title{
Urban Freight Management with Stochastic Time-Dependent Travel Times and Application to Large-Scale Transportation Networks
}

\author{
Shichao Sun, Zhengyu Duan, and Dongyuan Yang \\ Key Laboratory of Road and Traffic Engineering of the Ministry of Education, Tongji University, 4800 Cao'an Road, \\ Jiading District, Shanghai 201804, China
}

Correspondence should be addressed to Zhengyu Duan; d_zy@163.com

Received 10 July 2014; Accepted 6 October 2014

Academic Editor: Yongjun Shen

Copyright (C) 2015 Shichao Sun et al. This is an open access article distributed under the Creative Commons Attribution License, which permits unrestricted use, distribution, and reproduction in any medium, provided the original work is properly cited.

\begin{abstract}
This paper addressed the vehicle routing problem (VRP) in large-scale urban transportation networks with stochastic timedependent (STD) travel times. The subproblem which is how to find the optimal path connecting any pair of customer nodes in a STD network was solved through a robust approach without requiring the probability distributions of link travel times. Based on that, the proposed STD-VRP model can be converted into solving a normal time-dependent VRP (TD-VRP), and algorithms for such TD-VRPs can also be introduced to obtain the solution. Numerical experiments were conducted to address STD-VRPTW of practical sizes on a real world urban network, demonstrated here on the road network of Shenzhen, China. The stochastic timedependent link travel times of the network were calibrated by historical floating car data. A route construction algorithm was applied to solve the STD problem in 4 delivery scenarios efficiently. The computational results showed that the proposed STDVRPTW model can improve the level of customer service by satisfying the time-window constraint under any circumstances. The improvement can be very significant especially for large-scale network delivery tasks with no more increase in cost and environmental impacts.
\end{abstract}

\section{Introduction}

Urban freight distribution/delivery usually leads to traffic congestion, safety concerns, air pollution, and high logistic costs [1]. In recent years, more and more carriers and shippers have recognized the importance of designing efficient distribution strategies to improve the level of customers service and reduce the financial and environmental cost of freight transportation [2]. However, the extensive literature on the classical vehicle routing problem (VRP) and its variants have primarily considered the problem using static traffic information with corresponding constant travel times. In recent years, a number of studies take account of substantial variation in speeds and improve the model by taking the time dependency of travel times into consideration (see details in Section 2). However, in urban transportation system, lots of random factors, such as uncertain traffic volume, severe weather conditions, and incidents, can lead to the uncertainty of travel times during most of the day, especially during the morning and evening peak periods. Those nonrecurrent events can significantly affect the reliability of the transportation system and contribute to a stochastic timedependent (STD) congested transportation network. Urban route designs that ignore these significant variations and uncertainties of travel times are often found to be inefficient within a congested traffic condition and may contribute to higher operational costs or inferior customer service [1].

Therefore, in order to optimize the freight distribution performance in urban settings, both the random and timevarying properties of the link travel times must be considered. In this paper, we refer to the stochastic time-dependent VRP with hard time window (STDVRPTW). The aim of this study is to devise good and computationally efficient approaches to assist the fleet dispatchers operating in an urban congested environment. To utilize available resources for serve timesensitive customers, this paper takes into consideration of 
the following travel-time properties: (1) for certain routes, the travel times vary according to the time of day; (2) the travel time is stochastic.

The contributions of this paper are as follows.

(i) Taking account of the random and time-varying characters of traffic conditions, a robust optimization based method is applied in the proposed model, which is able to compute the stochastic timedependent optimal paths (STDOP) connecting any pair of customer nodes efficiently. Being different from many existing approaches, the robust approach does not require the probability distributions of link travel times and only takes the range of uncertainty which can be derived from historical data and experience of the decision-makers.

(ii) The STDVRP model we proposed here can be converted into a time-dependent VRP (TDVRP). The simplified problem will not lead to an increase in the computing time and can be solved efficiently by conventional algorithms. The proposed model is capable of addressing STDVRPTW of practical sizes on a real-world urban network, demonstrated here on the road network of Shenzhen, China, with computational instances of up to 150 customers.

(iii) The model we proposed can improve the level of customer service by guaranteeing the time-window constraint satisfied without leading to cost increase or environmental impacts. The improvement can be significant especially for large-scale network delivery tasks.

The rest of paper is organized as follows. In Section 2, a literature review is given on both VRP and optimal path problem in STD networks. In Section 3, the STD network is defined and the model of STDVRPTW is formulated. In Section 4, a routing construction algorithm is presented. Computational instance and analysis are shown in Section 5. In Section 6, conclusions are made and future directions are given.

\section{Literature Review}

This literature review is split into two parts: firstly, the literature related to the vehicle routing problems in terms of time dependency and randomness of link travel times is overviewed and secondly, the research on optimal path problem in STD networks is discussed.

2.1. Vehicle Routing Problem. Vehicle assignment and routing problems have been studied for several decades. Most traditional methodologies for this class of problems have been proposed based on adaptations of static algorithms and developed under static travel time, but they less consider dynamic traffic flow conditions [3].

As an extension of VRP, in order to consider possible variations of travel times in the network, Dynamic Vehicle Routing Problem is proposed. Malandraki and Daskin [4] put forward a strict mathematical model for TDVRP. They treat the travel time between two customers as a function of distance and the time of the day and design a nearest-neighbor heuristic algorithms and a cut plane heuristic algorithm. Ichoua et al. [5] introduce a First in First out (FIFO) principle into TDVRP and use time dependent function of speed to represent dynamic network, which avoids the deficiency of waiting in customer node in Malandraki and Daskin's model [4]. Donati et al. [6] extend this line of research by indicating the importance of optimizing the starting time and designing a more efficient heuristic algorithm in a time-dependent environment Recently, many research works adopt the timedependent speed assumption proposed in [5]. Using queuing models for time-dependent speeds, analytical expressions for the expected travel times as well as for the variance of the travel times are derived in [7]. A continuous function model of time-dependent link speeds is proposed in [8]. More realistic time-dependent information is obtained from archived historical travel data, as seen in [9]. A general modeling framework with finer time-dependent traffic information and efficient solution algorithm are proposed in [1] and are applicable to large-scale real world.

In this paper, we consider the VRP with dynamic and stochastic travel times (STDVRP), while there is limited research related to this topic. Lecluyse et al. [10] address the STDVRP by capturing the uncertainty in an analytical way using queuing theory and introduced the variability in traffic flows into the model, which allows for an evaluation of the routes based on the uncertainty involved. Nahum and Hadas [11] combine two important variants to form and define the STDVRP. Two algorithms for solving the stochastic time-dependent VRP are compared. As Ichoua et al. [5] indicate, stochastic and time-dependent travel times are more extensively operated on optimal path analysis between two service nodes when executing VRP delivery (see details in Section 2.2). Although mean and variance contain the most important information about path travel time, finding the single route with expected shortest travel time is not appropriate for routing when planners are not risk neutral. So in order to take account of the STD travel times in STDVRPTW, the optimal path problem in STD networks should be addressed as a subproblem first.

2.2. Optimal Path Problem in STD Networks. In realworld transportation networks, there exists more than one path connecting the current customer-node with the next unserved one, so taking which path to continue the delivery should be decided based on certain optimality criterions. That is to say, optimal path finding problem between two customer nodes is the fundamental subproblem for VRP and should be addressed first here to cope with the STD nature of travel times. If the uncertainty of link travel times can be captured to determine the optimal path efficiently, approaches may be more easily obtained for STDVRPTW. However, "optimal routes selection" in STD networks is more difficult than in deterministic networks, in part because, for a given departure time, more than one path may exist between an origin and destination, each with a positive probability of having the 
least travel time, so the definition of an optimal path can be somewhat indeterminate.

Hall [12] first puts forward the stochastic and timedependent optimal path problem (STDOPP). He chooses minimum expected travel time (METT) as the optimality criterion and proposes a branch-and-bound procedure for finding the METT path in STD networks. Miller-Hooks and Mahmassani explore the definition of optimality based on first-order stochastic dominance and definite dominance. After the 1990s, the utility theory in economics has been introduced to solve the STD optimal path problem. Wellman [13] identifies a stochastic consistent condition and presents a revised path-planning algorithm based on utility function. Huang and Gao [14] define a disutility function of travel time to evaluate the STD paths. They design an exact labelcorrecting algorithm to find the optimal path with the minimum expected disutility, but the algorithm had exponential computation complexity.

Most existing approaches to this problem in terms of different criterions of optimality rely on the precise probability distributions for link travel times, which is hard to realize in practical application. High computation complexity and inefficient algorithms are also strong restraints when solving large size network problems. In recent years, considering the "worst-case performance" of each path, the robust optimization theory has emerged as a preemptive way to address the uncertainties of link travel times without requiring exact probability distributions. Bertsimas and Sim [15] propose a linear robust optimization approach based on polyhedral uncertainty sets. Sim [16] proposes a new methodology to solve the stochastic optimal path problem, which promises greater computational tractability, both theoretically and practically, than the classical robust framework; however, he does not conduct a further study for solving the optimal path problem in STD networks.

2.3. Summary of Past Literature. Although routing models in STD networks are gaining greater attention in the literature and industry, a general modeling framework and efficient solution algorithm which is applicable to large-scale real-world networks are still lacking. Stochastic and timedependent travel times are more extensively operated on optimal path analysis between two service nodes when executing VRP delivery. However, most of the existing approaches to the STDOPP generally need a precise probability distribution of the uncertain link travel times which is hard to realize in practical application. High computation complexity and inefficient algorithms are also strong restraints when solving large size networks problems. In recent years, robust optimization theory has emerged as a preemptive way to address the uncertainties of link travel times with better computational tractability, meanwhile without requiring the precise probability distribution of link travel times.

In this paper, for such delivery routing with rigid arrival time requirements, the worst-case travel times of each candidate path connecting any pair of customer nodes should be considered to guarantee the time window constraint satisfied. Beyond that, the reliability of travel time is also a concern in delivery. So we refer to the robust approach here and apply it to solve the subproblem of STDVRPTW. The path, which minimizes the worst-case travel times over all the candidate paths, is defined as the optimal path connecting any two customer nodes.

\section{Model for STDVRPTW}

3.1. The Stochastic Time-Dependent Network. Given a directed graph $G=\left(N, A, T, C_{i j}^{t}\right)$, where $N$ is the set of the nodes and $A$ is the set of the links. The number of the nodes and links are denoted, respectively, as $|N|=n$ and $|A|=m$. T is the set of time intervals $\{0,1, \ldots, M-1\}$. Link $(i, j)$ represents the directed link from node $i$ to node $j$. A path connecting any pair of nodes can be denoted as a sequence of nodes. The travel time on link $(i, j)$ at time interval $t$ is denoted as $C_{i j}^{t}$. SR represents the set of links with random travel times. In this paper, it is assumed that all the link travel times in the network are random and dynamic, so $|\mathrm{SR}|=|A|=m$. Waiting time is not permitted at the upstream node $i$ before moving to the downstream node.

We define the travel time on link $(i, j)$ as

$$
C_{i j}^{t}=R_{i j}^{t}+\tau_{i j}^{t} .
$$

$R_{i j}^{t}$ is a fixed travel time value at time interval $t$ and $\tau_{i j}^{t}$ is a random variable ranging from 0 to $d_{i j}^{t}$, where $d_{i j}^{t}$ is also a fixed value at time interval $t$. Hence $C_{i j}^{t}$ is random time-dependent variable and takes value within $\left[R_{i j}^{t}, R_{i j}^{t}+d_{i j}^{t}\right]$.

Furthermore, it is assumed that the network model satisfies the stochastic consistent condition (SCC) proposed by Wellman [13]. That is, the network is stochastically consistent, if for any link $(i, j)$, at any time interval $t<t^{\prime}$ and any given time $z$, the following inequality holds:

$$
\operatorname{Pr}\left(C_{i j}^{t}+t \leq z\right) \geq \operatorname{Pr}\left(C_{i j}^{t^{\prime}}+t^{\prime} \leq z\right) .
$$

The inequality means that the probability of arriving by any given time $z$ cannot be increased by leaving later. Although overtaking exists in transportation network, such deterministic consistency condition (FIFO) property generally holds [14].

3.2. Mathematical Formulation. In this paper, we study STDVRP with hard time windows. It is assumed that there is only one depot from where the routes start and end for each vehicle, a homogeneous fleet consisting of several vehicles with fixed capacity, while the customers' demands are predetermined and each customer must be served within its time window. Unlike in manufacturing settings where buffer space may not be available in production facilities for goods arriving early, this paper permits early vehicles to wait till the beginning of time windows, as seen in Figure 1. Since STDVRP is a multiobjective optimization problem, this paper mainly considers the number of vehicles out for service and total schedule time, allowing different costs associated with each one. Beyond that, we also take into account that how many customer nodes failed to be served within their 


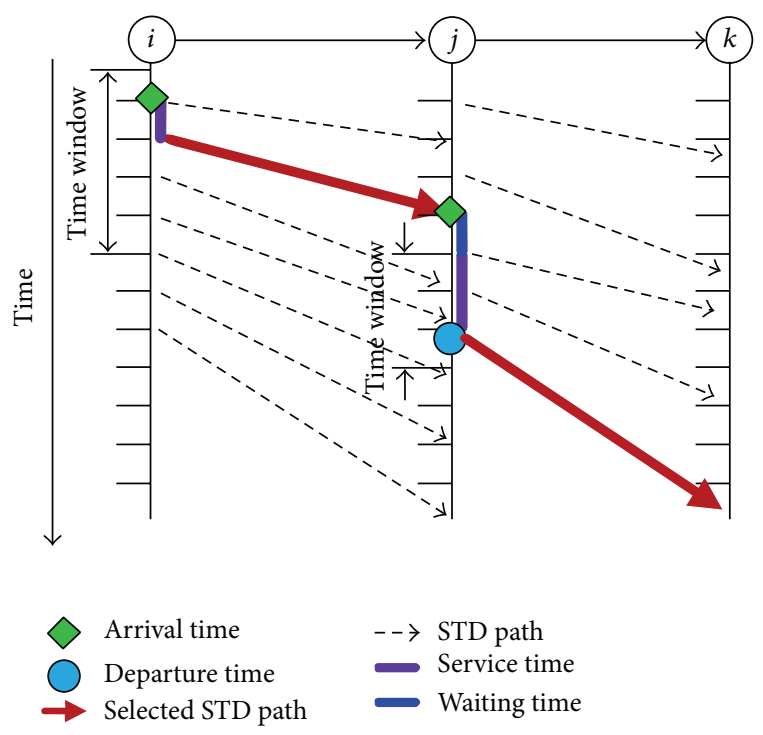

FIgURE 1: An Illustration of STDVRPTW. inequality always holds, it must have the following relation, which holds for any time interval $m_{2}<m_{2}^{\prime}$ :

$$
\begin{aligned}
R_{a b}^{m_{2}} & +\tau_{a b}^{m_{2}}+m_{2} \leq \max \left(R_{a b}^{m_{2}}+\tau_{a b}^{m_{2}}+m_{2}\right) \\
& =R_{a b}^{m_{2}}+d_{a b}^{m_{2}}+m_{2} \leq R_{a b}^{m_{2}^{\prime}}+d_{a b}^{m_{2}^{\prime}}+m_{2}^{\prime} .
\end{aligned}
$$

In this paper, each candidate path connecting any pair of costumer nodes can be denoted as a sequence of STD links. So the largest or worst-case travel times of the path can be obtained by calculating the travel times link by link. Furthermore, as the bold italic types shown in (4), it indicates that the worst-case travel times of each STD link satisfies the FIFO property, for example, link $(a, b)$. So if all the STD links take the worst-case value at the departing time of upstream nodes, it can guarantee the calculated path travel time being the largest. Then we can obtain the worst-case travel times for each candidate path, as shown in (5) as follows:

$$
\begin{gathered}
\max _{\text {for each candidate path }} \sum_{(a, b) \in A} \sum_{m_{2}=1}^{M}\left(R_{a b}^{m_{2}}+\tau_{a b}^{m_{2}}\right) \cdot y_{a b}^{m_{2}} \\
=\sum_{(a, b) \in A} \sum_{m_{2}=1}^{M}\left(R_{a b}^{m_{2}}+d_{a b}^{m_{2}}\right) \cdot y_{a b}^{m_{2}}, \\
T_{i j}^{m_{1}}=\operatorname{Min}\left(\sum_{(a, b) \in A} \sum_{m_{2}=1}^{M}\left(R_{a b}^{m_{2}}+d_{a b}^{m_{2}}\right) \cdot y_{a b}^{m_{2}}\right) .
\end{gathered}
$$

In (6), $R_{a b}^{m_{2}}$ and $d_{a b}^{m_{2}}$ change along with the time-interval $m_{2} \cdot R_{a b}^{m_{2}}+d_{a b}^{m_{2}}$ is not the actual travel time but the upper bound of travel time on link $(a, b)$ at time-interval $m_{2}$, which satisfies FIFO property. So $T_{i j}^{m_{1}}$ can be solved as the shortest path problem in a FIFO network with time-dependent upper bound of link travel times $R_{a b}^{m_{2}}+d_{a b}^{m_{2}}$. Furthermore, the TD shortest path problem in FIFO networks has been proved to be solved in polynomial time, and the extended conventional labeling setting algorithms can be modified to solve the problem with no more computation complexity than that in a static network [17].

$$
T_{i j}^{m_{1}}=\operatorname{Min}\left(\underset{\text { Candidate Paths }}{\operatorname{Max}} \sum_{(a, b) \in A} \sum_{m_{2}=1}^{M}\left(R_{a b}^{m_{2}}+\tau_{a b}^{m_{2}}\right) \cdot y_{a b}^{m_{2}}\right) .
$$

In STD networks, according to the aforementioned stochastic consistent condition, for any link $(a, b)$, at any time interval $m_{2}<m_{2}^{\prime}$, and any given time $z$, we can get (2).

In (2), let $Z=\max \left(R_{a b}^{m_{2}^{\prime}}+\tau_{a b}^{m_{2}^{\prime}}+m_{2}^{\prime}\right)=R_{a b}^{m_{2}^{\prime}}+d_{a b}^{m_{2}^{\prime}}+m_{2}^{\prime}$. Then, it can be observed that the probability of the right-side formula of (2) is $100 \%$. Then we can determine that if the
3.2.3. Model for STDVRPTW. The following is a mixedinteger programming model for STDVRPTW.

Objective Function. Consider

$\min K$,

$\min \mathrm{ST}$ 
TABLE 1: Notations and variables.

\begin{tabular}{|c|c|c|}
\hline Type & Symbol & Definition \\
\hline \multirow{5}{*}{$\begin{array}{l}\text { Objective } \\
\text { function } \\
\text { quantities }\end{array}$} & $\mathbf{K}$ & The number of vehicles out for service \\
\hline & ST & Total schedule time \\
\hline & TT & Total travel time \\
\hline & WT & Total waiting time \\
\hline & SVT & Total service time \\
\hline \multirow{9}{*}{ Sets } & $S$ & Set of starting nodes of all vehicles \\
\hline & $\mathbf{E}$ & Set of end nodes of all vehicles \\
\hline & $\mathbf{D}$ & Set of all customer nodes \\
\hline & $\mathbf{i} \in \mathbf{S D}$ & Set of starting nodes and customer nodes \\
\hline & $\mathbf{j} \in \mathrm{DE}$ & Set of customer nodes and end nodes \\
\hline & $\mathbf{i}, \mathbf{j} \in \mathrm{SDE}$ & Set of all nodes \\
\hline & $\mathrm{C}$ & Set of all customers \\
\hline & $\mathrm{SC} \subseteq \mathrm{C}$ & Subset of set $\mathbf{C}$ \\
\hline & $\operatorname{Link}(\mathbf{a}, \mathbf{b}) \in \mathbf{A}$ & Set of links that are present in the networks \\
\hline \multirow{8}{*}{ Inputs and parameters } & $\mathbf{M}$ & The number of time intervals \\
\hline & $\mathbf{m}_{1}, \mathbf{m}_{2}=1,2, \ldots, \mathbf{M}$ & Time interval $\mathbf{m}_{1}, \mathbf{m}_{2}$ \\
\hline & B & An infinite number \\
\hline & svt $_{\mathbf{i}}$ & Service time of customer node $i$ \\
\hline & $\mathbf{q}_{\mathrm{i}}$ & Delivery demand of customer node $i$ \\
\hline & {$[\mathbf{0}, \mathbf{T}]$} & Time window of depot \\
\hline & {$\left[e_{i}, l_{i}\right]$} & Time window constraint of customer node $i$ \\
\hline & $\mathbf{Q}_{\mathrm{k}}$ & Capacity of vehicle $k$ \\
\hline \multirow{5}{*}{ Decision variables } & $\mathbf{t}_{\mathbf{i}}$ & Vehicle's arrival time at node $i$ \\
\hline & $y_{a b}^{m_{2}}$ & $\mathbf{y}_{\mathrm{ab}}^{\mathbf{m}_{2}}=1$, if any vehicle occupies link $(\mathbf{a}, \mathbf{b})$ at time $\mathbf{m}_{2}$; otherwise $\mathbf{y}_{\mathrm{ab}}^{\mathbf{m}_{2}}=0$ \\
\hline & $\mathrm{T}_{\mathrm{ij}}^{\mathrm{m}_{1}}$ & $\begin{array}{l}\text { Travel times of the optimal path from customer node } i \text { to customer node } j \text { at } \\
\text { departing time interval } \mathbf{m}_{\mathbf{1}}\end{array}$ \\
\hline & $\mathrm{x}_{\mathrm{ij}}^{\mathrm{m}_{1}}$ & $\begin{array}{l}\mathbf{x}_{\mathrm{ij}}^{\mathbf{m}_{1}}=\mathbf{1} \text {, if vehicle departs from customer node } i \text { to customer node } j \text { at time interval } \\
\mathbf{m}_{1} ; \text { otherwise } \mathbf{x}_{\mathbf{i j}}^{\mathbf{m}_{1}}=0\end{array}$ \\
\hline & $\mathbf{v}_{\mathbf{i k}}$ & $\mathbf{v}_{\mathbf{i k}}=1$, if customer node $i$ is visited by vehicle $k$; otherwise, $\mathbf{v}_{\mathbf{i k}}=0$ \\
\hline
\end{tabular}

Expressions of Objective Function. Consider

$$
\begin{aligned}
\mathrm{ST} & =\mathrm{TT}+\mathrm{WT}+\mathrm{SVT}, \\
\mathrm{TT} & =\sum_{i \in S D} \sum_{\substack{j \in D E \\
j \neq i}} \sum_{m_{1}=1}^{M}\left(T_{i j}^{m_{1}} \cdot x_{i j}^{m_{1}}\right), \\
\mathrm{WT} & =\sum_{i \in D} \max \left(e_{i}-t_{i}, 0\right), \\
T_{i j}^{m_{1}} & =\operatorname{Min}\left(\max _{\text {for each candidate path }} \sum_{(a, b) \in A} \sum_{m_{2}=1}^{M}\left(R_{a b}^{m_{2}}+\tau_{a b}^{m_{2}}\right) \cdot y_{a b}^{m_{2}}\right) \\
& =\operatorname{Min}\left(\sum_{(a, b) \in A} \sum_{m_{2}=1}^{M}\left(R_{a b}^{m_{2}}+d_{a b}^{m_{2}}\right) \cdot y_{a b}^{m_{2}}\right), \\
t_{i} & =\max \left(e_{i-1}, t_{i-1}\right)+\mathrm{svt}_{i-1}+T_{(i-1) i}^{m_{1}}, \\
\mathrm{SVT} & =\sum_{i \in D} \mathrm{svt}_{i} .
\end{aligned}
$$

\section{Constraints. Consider}

$$
\begin{gathered}
\sum_{\substack{i \in S D \\
i \neq j}} \sum_{m_{1}=1}^{M} x_{i j}^{m_{1}}=1, \quad j \in D E, \\
\sum_{\substack{j \in D E \\
i \neq j}} \sum_{m_{1}=1}^{M} x_{i j}^{m_{1}}=1, \quad i \in S D, \\
\sum_{k=1}^{K} k\left(v_{i k}-v_{j k}\right) \leq B\left(1-\sum_{m_{1}=1}^{M} x_{i j}^{m_{1}}\right), \\
i \in S D, \quad j \in D E, \quad i \neq j, \\
\sum_{k=1}^{K} k\left(v_{i k}-v_{j k}\right) \geq B\left(\sum_{m_{1}=1}^{M} x_{i j}^{m_{1}}-1\right), \\
i \in S D, \quad j \in D E, \quad i \neq j,
\end{gathered}
$$




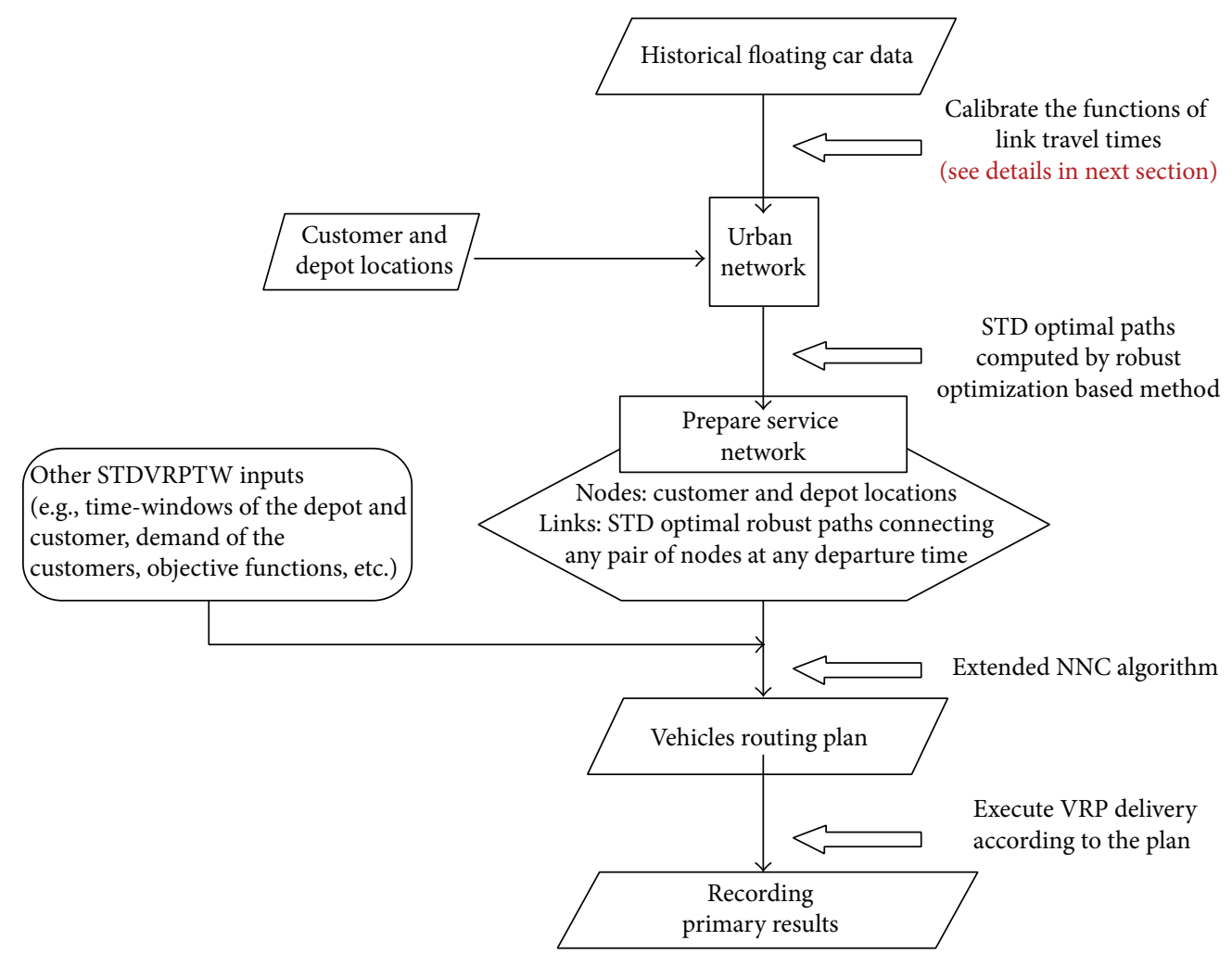

FIGURE 2: The illustrated solving procedure of STDVRPTW modeling.

$$
\begin{gathered}
\sum_{k=1}^{K} v_{i k}=1, \quad i \in D, \\
\sum_{i \in D}\left(q_{i} \cdot v_{i k}\right) \leq Q_{k}, \quad k=1,2,3,4, \\
\sum_{i \in s} \sum_{j \in D E} \sum_{m_{1}=1}^{M} x_{i j}^{m_{1}}=\sum_{i \in S D} \sum_{j \in D} \sum_{m_{1}=1}^{M} x_{i j}^{m_{1}}, \\
\sum_{\substack{i, j \in S C \\
i \neq j}} \sum_{m_{1}=1}^{M} x_{i j}^{m_{1}} \leq|S C|-1, \quad S C \in C, \\
\max \left(e_{i-1}, t_{i-1}\right)+s v t_{i-1}+T_{(i-1) i}^{m_{1}} \leq l_{i}, \quad i \in D, \\
t_{\text {depot }} \leq T, \quad t_{0}=0 .
\end{gathered}
$$

Objective function (7) minimizes the number of vehicles out for service. Objective function (8) minimizes the total schedule time (ST), where ST is the sum of total travel time (TT), total waiting time (WT), and total service time (SVT). Constraints (10) and (11) state that each customer can be served only once. Constraints (12)-(14) state that customers for each route can be served by only one vehicle. Constraint (15) states that the capacity of customers for each route does not exceed the maximum capacity of a single vehicle. Constraint (16) states that all the utilized vehicles should return to the depot. Constraint (17) is added for route continuity. Constraints (18) and (19) are added as time window constraints.

\section{Algorithm for STDVRPTW}

In the model of STDVRPTW that we proposed, the only dynamic random variable $T_{i j}^{m}$ has been converted into a timedependent function which can be computed by solving a shortest path problem with time dependent travel times (see details in Section 3.2.2, denoted as (6)). So the STDVRP can be simplified into a TDVRP, and algorithms for such TDVRPs can also be applied to obtain the solution. Since VRP is a NP-hard optimization problem, the complexity of the STD problem will remain the same as capacitated VRP at least, because of the time dimension and the stochastic properties of the problem. Such complexity calls for the development of an efficient heuristic algorithm.

Duan et al. [18] extended the route construction algorithm, namely, NNC (nearest neighbor algorithm base on minimum cost) algorithm for solving the TDVRP in extended Solomon instances. Considering computing time and solution quality synthetically, he proved that the extended NNC algorithm had a better performance compared with some other existing algorithms. The same algorithm is also applied in this paper to solve the simplified STDVRPTW.

\section{Case Study}

The main goals of this computational test are (a) to illustrate the importance of considering the STD characters of the transportation networks when designing the delivery route 
TABLE 2: Computational results and evaluation of routing plans in 4 delivery scenarios.

\begin{tabular}{|c|c|c|c|c|c|c|c|c|}
\hline Scenario & $\begin{array}{l}\text { Instance specification } \\
\text { Number of customers }\end{array}$ & Model & Vehicle number & Schedule time ${ }^{b}$ & Travel time & Failure number & $\begin{array}{l}\text { Failure } \\
\text { rate }^{c}\end{array}$ & CPU times (s) \\
\hline \multirow{3}{*}{1} & \multirow{3}{*}{25} & $\mathrm{TD}$ & 4 & 43.76 & 22.44 & 5.5 & $22 \%$ & 5.55 \\
\hline & & STD & 5 & 47.35 & 23.61 & 0 & 0 & 5.27 \\
\hline & & $\Delta^{\mathrm{a}}$ & $25.00 \%$ & $8.20 \%$ & $5.21 \%$ & None & $-22 \%$ & $-5 \%$ \\
\hline \multirow{3}{*}{2} & \multirow{3}{*}{50} & $\mathrm{TD}$ & 7 & 60.24 & 29.98 & 11.4 & $22.8 \%$ & 19.63 \\
\hline & & STD & 9 & 65.62 & 31.20 & 0 & 0 & 17.08 \\
\hline & & $\Delta$ & $28.57 \%$ & $8.93 \%$ & $4.07 \%$ & None & $-22.8 \%$ & $-13 \%$ \\
\hline \multirow{3}{*}{3} & \multirow{3}{*}{100} & TD & 15 & 116.97 & 47.98 & 20.3 & $20.3 \%$ & 71.11 \\
\hline & & STD & 17 & 126.89 & 53.25 & 0 & 0 & 66.58 \\
\hline & & $\Delta$ & $13.33 \%$ & $8.48 \%$ & $10.98 \%$ & None & $-20.3 \%$ & $-6 \%$ \\
\hline \multirow{3}{*}{4} & \multirow{3}{*}{150} & TD & 20 & 179.15 & 88.61 & 31.4 & $20.9 \%$ & 148.38 \\
\hline & & STD & 23 & 202.99 & 98.08 & 0 & 0 & 137.88 \\
\hline & & $\Delta$ & $15.00 \%$ & $13.31 \%$ & $10.69 \%$ & None & $-20.9 \%$ & $-7 \%$ \\
\hline
\end{tabular}

${ }^{\mathrm{a}} \Delta$ indicates relative percentage change between the two tests.

${ }^{\mathrm{b}}$ Total schedule time is the sum of total travel time (TT), total waiting time (WT), and total service time (SVT).

${ }^{\mathrm{c}}$ Failure rate $=$ failure number/number of customers.

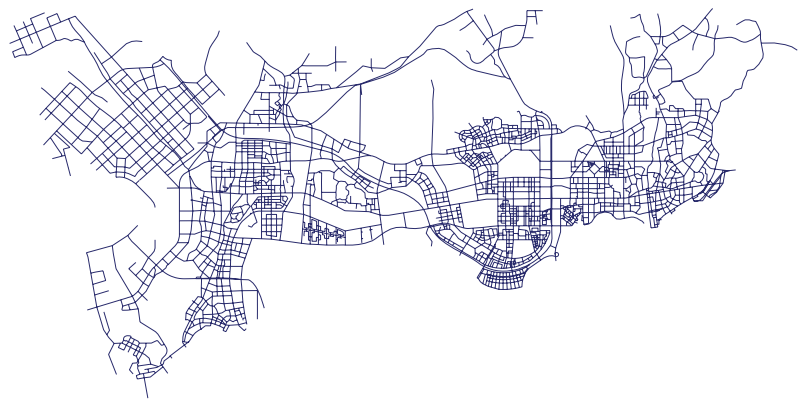

FIgURE 3: The transportation network of Shenzhen, China.

plans and (b) to confirm the validity of robust approach, by use of which, the STD optimal paths connecting any pair of customer nodes can be computed, the VRP model can be simplified (STDVRPTW to TDVRPTW), and it is also able to gain a high service-level solution without increasing the computing time, and (c) to verify that the solution algorithm works well in a simulated large-scale urban transportation network with STD link travel times for an entire planning horizon. The functions of link travel times in the test network can be calibrated by the use of historical floating car data which can be derived from taxis equipped with GPS devices. The solving procedure can be illustrated in Figure 2.

\subsection{Construction of Test Instance in Real Urban Transportation Network}

\subsubsection{Experimental Setting}

(1) The Large-Scale Urban Road Network. Numerical experiments were conducted on the urban transportation network of Shenzhen, China, consisting of 3,454 nodes and 4,876 links, shown in Figure 3. The network covers the downtown area of Shenzhen.

(2) Stochastic Time-Dependent Link Travel Times of the Network Calibrated by Historical Floating Car Data. Floating car data (FCD) collection system is a new traffic detection technology in recent years, which uses the position data transmitting from on-board GPS equipment in regular interval, to compute the traffic parameters of speed and travel time and so forth and get the real-time traffic condition of the network. Compared with fixed-point traffic detector like inductive loop and microware radar, it is advantageous in wide coverage, high efficiency, and low operation cost.

In this paper, the historical floating car data (travel speeds) were obtained at a 5-minute interval (total 288 intervals for one day) from over 5,000 taxis equipped with FCD collection devices in Shenzhen, China, during workdays in one week. After raw data filtering, recovering, and mapmatching processing, a set of discrete travel speeds on each link at a 5-minute interval can be obtained. Then for each link of the network, the upper and lower bound of obtained link travel speeds at each interval can be calibrated by the maximum and minimum value of the processed data; moreover, in order to reflect the randomness of the network, the link travel speed at each interval was assumed to take values randomly within the ranges (hypothetical Gaussian distribution). So the time-dependent step function of stochastic link travel speeds can be established with 288 intervals, through which the stochastic time-dependent link travel times of the network can be obtained. The calibration procedure is illustrated in Figure 4.

(3) Travel Times of the STD Optimal Path Connecting Any Pair of Customer Nodes. Since link travel times of the network have been calibrated at 288 intervals. Travel times of the STD optimal paths connecting any pair of customer nodes can be 


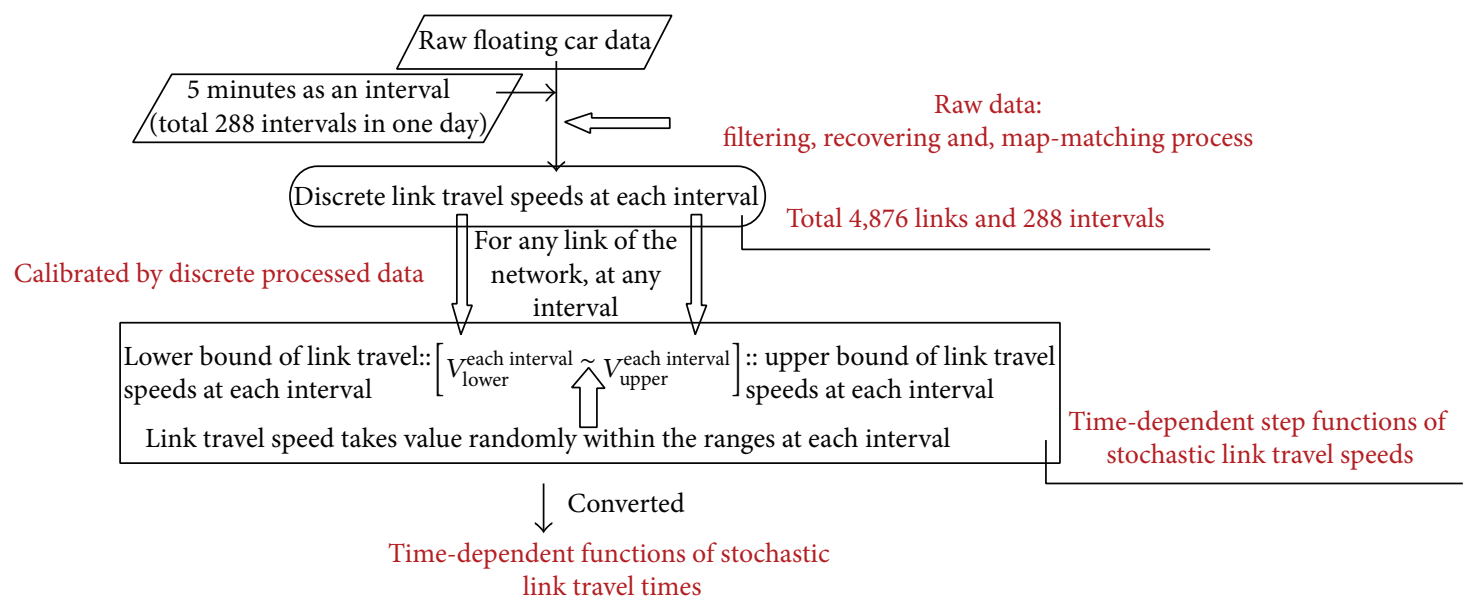

FIGURE 4: The calibration procedure of stochastic time-dependent travel times.

computed at each interval by the proposed robust approach in Section 3.2.2.

(4) Depot and Customer Nodes Information. Four delivery scenarios with different number of customer nodes were set for the test. The spatial distribution of depot and customer nodes is shown in Figure 5. The symbol $\star$ denoted depot, and - denoted customer node.

\section{(5) Customer Information}

(i) Demand range: 1-40 units (random integers drawn from the Solomon's test instance R101 [19]).

(ii) Service time of customers: the service time of each customer is fixed as 30 minutes.

(iii) The earliest service time of each customer: the earliest start service time of each customer is generated based on the test instance R101 of Solomon instances [19], ranging from $8 \mathrm{AM}$ to $5 \mathrm{PM}$.

\section{(6) Time Window Information}

(i) The working hours of the depot is [8:00, 19:00].

(ii) 30 minutes time window of customers: specify 30 minutes time window from the earliest start service time for each of the customers.

\section{(7) Vehicle Information}

(i) Fleet size is 30 .

(ii) Vehicle capacity is 200 units.

5.1.2. Test Environment. The test environment is as follows:

(i) CPU is Intel Core i3 (Quad-Core), 2.93 GHz.

(ii) Memory is $4 \mathrm{~GB}$.

(iii) Operation Systems is Windows 2003 Server.

(iv) Program Language is Microsoft C\# .Net.
5.2. Computational Results. The STDVRPTW were solved, respectively, in the 4 delivery scenarios on the large-scale road network of Shenzhen, China, by the extended NNC algorithm. Meanwhile, in order to test the effectiveness of the approach we proposed, another TDVRPTW test was conducted as the reference in the same instances (the expected travel times were used to cope with the fluctuations of travel time in STD instances for a priori delivery-route planning; therefore it can be considered as a TDVRP). In these two tests, the a priori delivery-route plans in the instances were first generated based on the model of STDVRPTW and TDVRPTW. Then the delivery task was executed according to the a priori plans, during which the link travel times were generated randomly (hypothetical Gaussian distribution) between the upper and lower bound of the calibrated travel times. The number of vehicles out for service, total schedule times, and CPU times were calculated, respectively, in the tests. The indicator failure number denoted how many customer nodes failed to be served within their time windows due to the fluctuation of travel times during the actual execution (if a vehicle arrives at the customer node beyond its upper bound of time window, the service will not start and the vehicle will continue its next delivery node according to the plan). The comparative results between STDVRPTW and TDVRPTW are shown in Table 2.

5.2.1. Comparison of Primary Results and Evaluation of Routing Plans. Table 2 shows a comparison of the results obtained from all instances. As expected, the total cost in STDVRPTW test, including the number of vehicles out for service and the total schedule times, is a little higher compared with the counterpart instances in TD situation. The relative increase of cost regarding the total schedule time can go up to $13.31 \%$ in Scenario 4. Similar tendency is also observed for the total number of vehicles; that is, STD model requires more vehicles to serve all customer nodes, but with the number of customers increasing in large-scale delivery scenarios (Scenarios 3 and 4), the relative percentage of increase becomes smaller. However, if we take the failure 

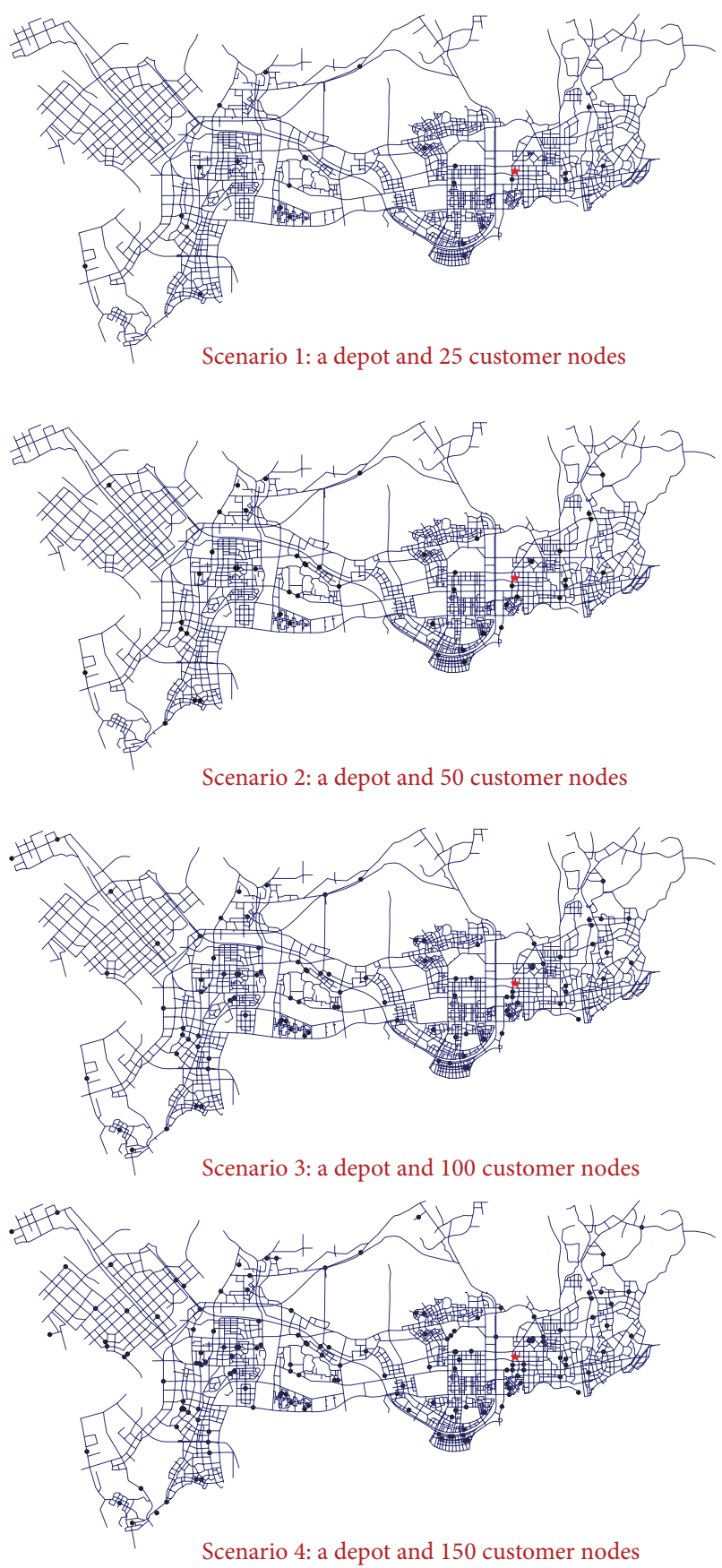

FIgURE 5: Spatial distribution of depot and customer nodes on the network (4 scenarios).

number of service into consideration, the cost gap may be ignored, which will be explained in the following.

The comparison result regarding the number of customer nodes that fail to be served within time windows in actual execution is quite the contrary. The failure rates of TD model in 4 delivery scenarios always retain larger than $20 \%$ and seem to be invariant with the number of customers. However, there exist no delivery failures in the actual delivery executions when using our STDVRP model, as shown in Table 2. Such comparison results can be explained as follows: the expected travel times are used in TDVRP test to cope with the fluctuations of travel times for a priori route planning. However, the actual travel times in STD networks are generated randomly between the upper and lower bounds of the calibrated travel times during the execution of delivery, so it cannot guarantee the actual travel times satisfying the time-window constraint of next customer node and may lead to the failure of delivery task. In contrast, it can be entirely avoided in the model of STDVRPTW we proposed by considering the worst-case travel times though robust approach.

The good news is that the relative percentage increase of the total cost seems to become smaller with the increasing number of the customers, while the failure rate in TD model always retains a high $20 \%$ level. Since the fail-serviced customer nodes in real life generally need to be served again afterwards, with the network scale expanding, more extra vehicles and operational costs would be paid on the basis of first round service. Thus, it indicates that for large-scale network delivery task, the improvement of service level can be very significant by use of our STDVRP model at no more expense of increases in cost and environmental impacts.

5.2.2. Comparison of CPU Time. From the statistics in Table 2, we can find that the STDVRPTW in 4 scenarios requires more or less the same computational time as the counterparts in TDVRP, and both of them can be solved efficiently in large-scale delivery scenario by NNC algorithm (137.88 s in 150 customers situations by use of STD model). It implies that the VRPTW in STD networks can be solved as efficiently as TDVRPTW by the NNC algorithm and may have a good potential of application in large-scale network.

\section{Conclusion and Future Directions}

This paper addressed the VRPTW of practical sizes on a realworld network with STD travel times. A robust optimization based method was proposed to solve the STD optimal path problem between any pair of customer nodes, which is considered as the subproblem of STDVRPTW, and the path which minimizes the worst-case travel time over all the candidate paths is defined as the optimal. With the subproblem solved, the STDVRPTW can be simplified into a TDVRP and algorithms for such TDVRPs can also be introduced to obtain the solution. Numerical experiments were conducted on the urban transportation network of Shenzhen, China, consisting of 3,454 nodes and 4,876 links. The stochastic timedependent link travel times of the network were calibrated at 288 intervals by the use of historical floating car data. Then the NNC algorithm was applied to solve the problem in 4 delivery scenarios. The computational results showed that the proposed STDVRPTW model can improve the level of customers service by guaranteeing the time-window constraint satisfied. The improvement can be very significant especially for large-scale network delivery tasks at no more expense of increases in cost and environmental impacts.

We would like to continue the work on analyzing STDVRP in large-scale transportation networks using real-time information. More computational tests can be conducted 
to analyze the potential of our approach in practice. More algorithms, such as Genetic Algorithm and Ant Colony Algorithm, will be applied in future to obtain better solutions.

\section{Conflict of Interests}

The authors declare that there is no conflict of interests regarding the publication of this paper.

\section{Acknowledgment}

This research is supported by the National Natural Science Foundation of China (no. 71001079). The authors would like thank their teammates for their time, suggestions, and valuable comments.

\section{References}

[1] L. Jiang and H. S. Mahmassani, City Logistics: Freight Management with Time Dependent Travel Times and Application to Large-Scale Networks, CD-ROM, Transportation Research Board of the National Academies, Washington, DC, USA, 2013.

[2] T. G. Crainic, N. Ricciardi, and G. Storchi, "Models for evaluating and planning city logistics systems," Transportation Science, vol. 43, no. 4, pp. 432-454, 2009.

[3] T.-Y. Liao and T.-Y. Hu, "An object-oriented evaluation framework for dynamic vehicle routing problems under real-time information," Expert Systems with Applications, vol. 38, no. 10, pp. 12548-12558, 2011.

[4] C. Malandraki and M. S. Daskin, "Time dependent vehicle routing problems: formulations, properties and heuristic algorithms," Transportation Science, vol. 26, no. 3, pp. 185-200, 1992.

[5] S. Ichoua, M. Gendreau, and J.-Y. Potvin, "Vehicle dispatching with time-dependent travel times," European Journal of Operational Research, vol. 144, no. 2, pp. 379-396, 2003.

[6] A. V. Donati, R. Montemanni, N. Casagrande, A. E. Rizzoli, and L. M. Gambardella, "Time dependent vehicle routing problem with a multi ant colony system," European Journal of Operational Research, vol. 185, no. 3, pp. 1174-1191, 2008.

[7] T. van Woensel, L. Kerbache, H. Peremans, and N. Vandaele, "A queueing framework for routing problems with timedependent travel times," Journal of Mathematical Modelling and Algorithms, vol. 6, no. 1, pp. 151-173, 2007.

[8] A. Haghani and S. Jung, "A dynamic vehicle routing problem with time-dependent travel times," Computers and Operations Research, vol. 32, no. 11, pp. 2959-2986, 2005.

[9] R. G. Conrad and M. Figliozzi, "Algorithms to quantify impact of congestion on time-dependent real-world Urban Freight distribution networks," Transportation Research Record, vol. 2168, pp. 104-113, 2010.

[10] C. Lecluyse, T. Van Woensel, and H. Peremans, "Vehicle routing with stochastic time-dependent travel times," 4OR. A Quarterly Journal of Operations Research, vol. 7, no. 4, pp. 363-377, 2009.

[11] O. E. Nahum and Y. Hadas, "A comparison of two algorithms for the stochastic time-dependent vehicle-routing problem," in Proceedings of the Transportation Research Board 89th Annual Meeting, Washington, DC, USA, 2010.

[12] R. W. Hall, "The fastest path through a network with random time-dependent travel times ," Transportation Science, vol. 20, no. 3, pp. 182-188, 1986.
[13] M. P. Wellman, "Path planning under time-dependent uncertainty," in Proceedings of the 11th Conference on Uncertainty in Artificial Intelligence, pp. 18-20, Montreal, Quebec, Canada, 1995.

[14] H. Huang and S. Gao, "Optimal paths in dynamic networks with dependent random link travel times," Transportation Research Part B: Methodological, vol. 46, no. 5, pp. 579-598, 2012.

[15] D. Bertsimas and M. Sim, "Robust discrete optimization and network flows," Mathematical Programming, vol. 98, no. 1-3, pp. 49-71, 2003.

[16] M. Sim, Robust optimization [Ph.D. Dissertation], Massachusetts Institute of Technology, Cambridge, Mass, USA, 2004, http://course.shufe.edu.cn/jpkc/jcjx/zyff/doc/tl11.pdf.

[17] D. E. Kaufman and R. L. Smith, "Minimum travel time paths in dynamic networks with application to intelligent vehicle/ highway systems," IVHS Journal, vol. 1, no. 1, pp. 1-19, 1993.

[18] Z. Duan, D. Yang, W. Sun, and S. Wang, "Route construction algorithms for time dependent vehicle routing problem," in Proceedings of the International Conferen ce of Logistics Engineering and Management, pp. 3467-3474, Chengdu, China, October 2010.

[19] M. Solomon, “VRPTW Benchmark Problems," http://w.cba.neu .edu/ msolomon/problems.htm. 


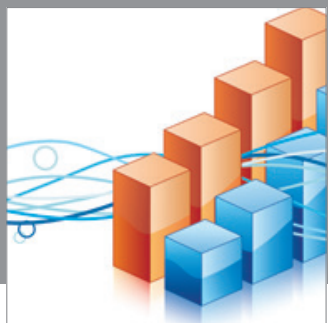

Advances in

Operations Research

mansans

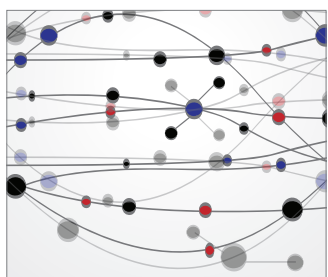

The Scientific World Journal
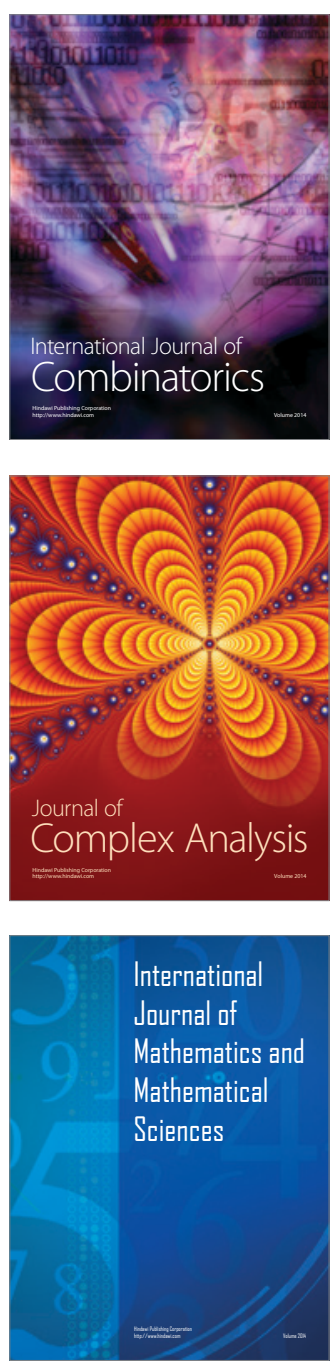
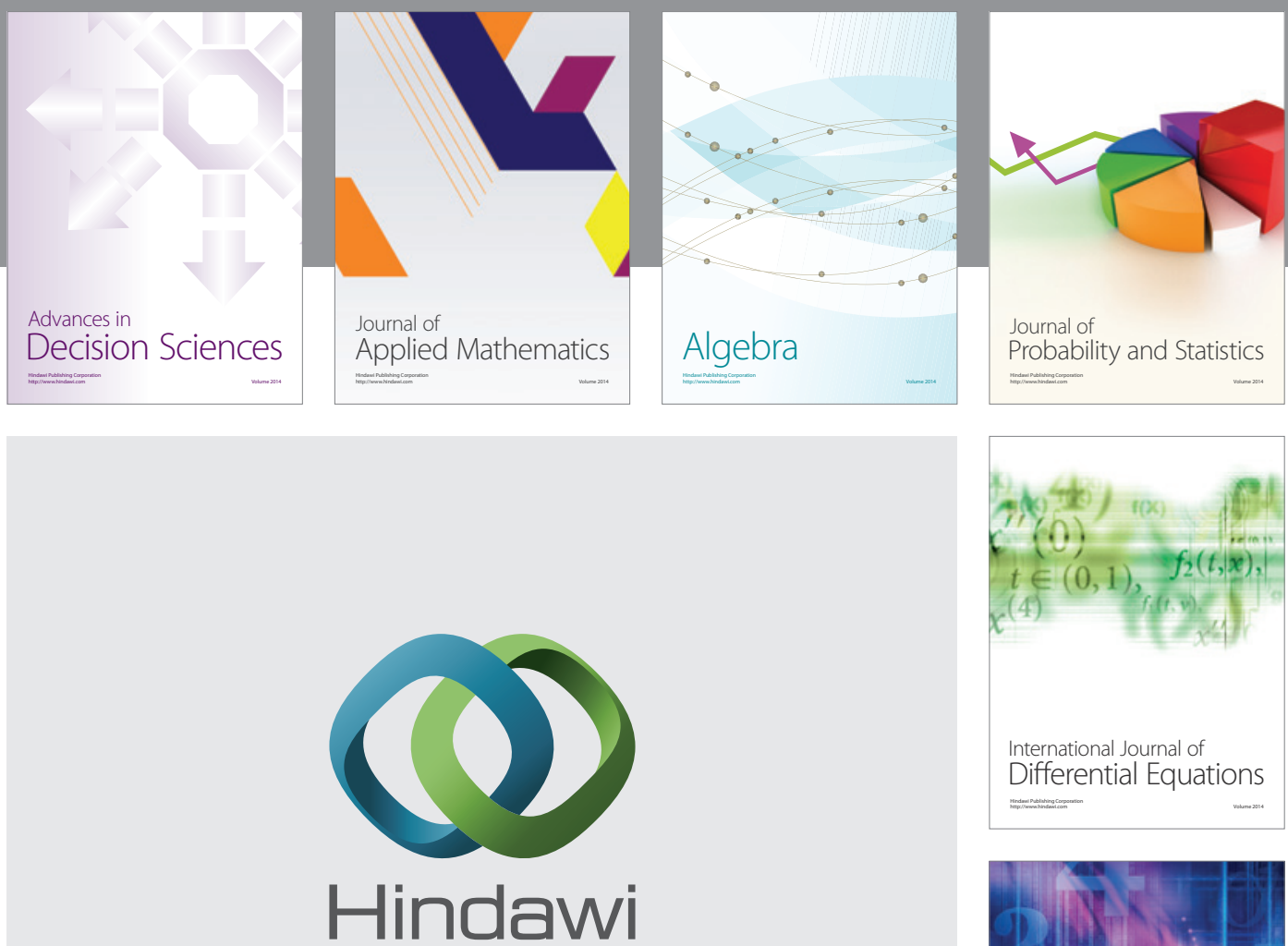

Submit your manuscripts at http://www.hindawi.com
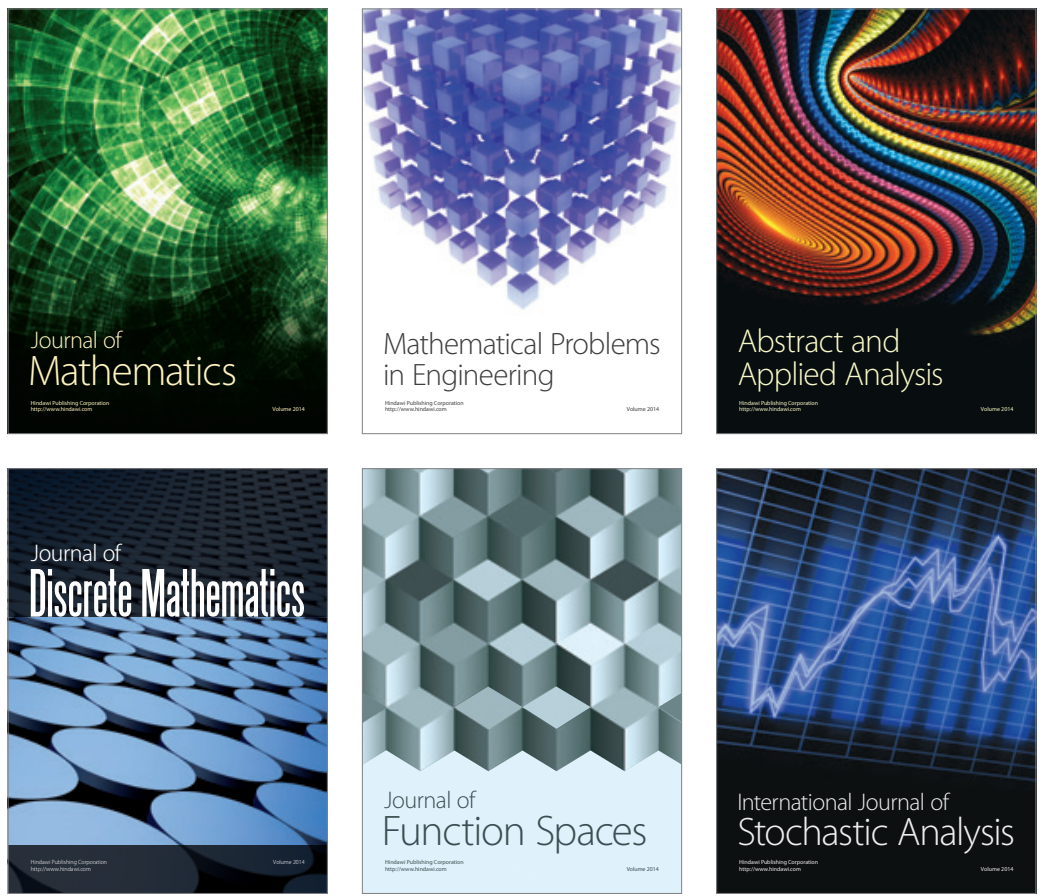

Journal of

Function Spaces

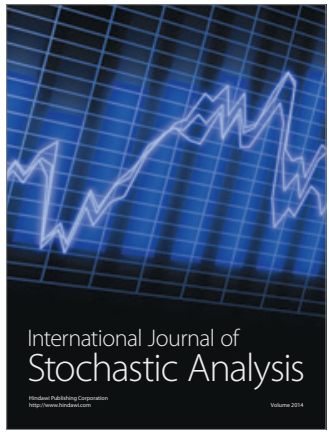

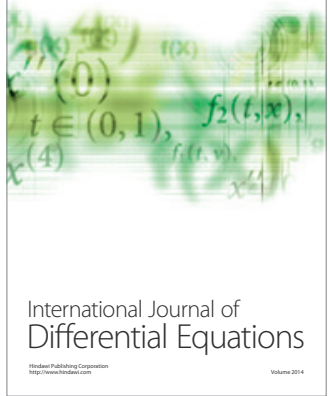
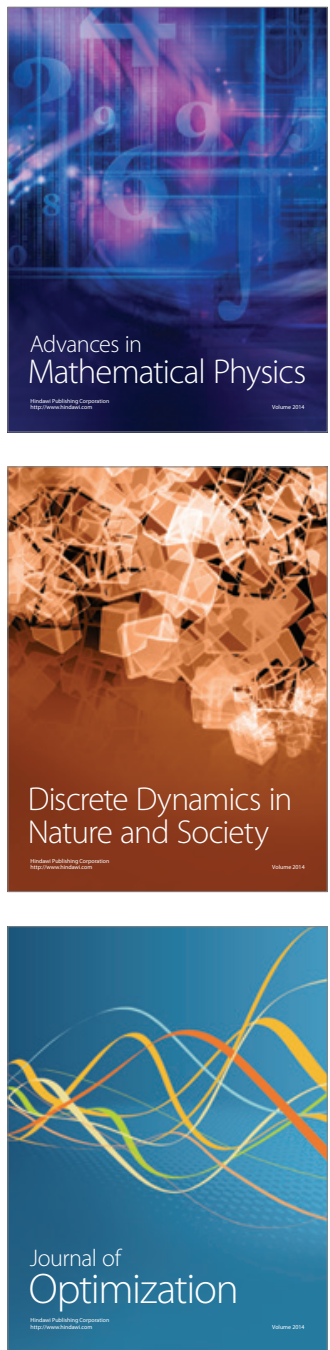